\title{
Amine content of vaginal fluid from patients with trichomoniasis and gardnerella associated non- specific vaginitis
}

\author{
B E SANDERSON,* E WHITE,* AND M J BALSDON† \\ From the *Research Institute, May \& Baker Ltd, Dagenham, Essex and the +Department of \\ Genitourinary Medicine, St Mary's Hospital, Portsmouth
}

SUMMARY Amounts of putrescine, cadaverine, and tyramine were measured in vaginal washings from five patients with non-specific vaginitis (NSV) associated with Gardnerella vaginalis, five patients with trichomoniasis, and five healthy controls. Putrescine and cadaverine were present in all but one sample from the infected patients; tyramine was found in four of five samples from the women with NSV and in two of five samples from those with trichomoniasis. The mean cadaverine to putrescine ratio was significantly higher in the patients with NSV than in those with trichomoniasis. Samples from the five controls contained only very small amounts of any of the three amines. The amine content of nine of 10 samples from the infected women was reduced to normal values after metronidazole treatment. The possible sources of the amines from Trichomonas vaginalis and Gardnerella vaginalis are discussed with reference to the clinical response to treatment.

\section{Introduction}

Vaginal washings from patients with non-specific vaginitis (NSV) associated with Gardnerella vaginalis contain several amines, the most abundant being putrescine and cadaverine. ${ }^{1}$ These amines may be produced mainly by anaerobic vaginal bacteria, which are present in greater numbers in patients with NSV than in normal women. ${ }^{2}$ Trichomonas vaginalis produces large amounts of putrescine in vitro (unpublished data), and recently diamines have been associated with this organism in the vagina. ${ }^{3}$ It has been suggested that a simple qualitative test for measuring diamines in the vaginal fluid is useful in the diagnosis of NSV or trichomoniasis. ${ }^{3}$

We measured the amounts of putrescine, cadaverine, and tyramine in vaginal washings from five women with NSV, five with trichomoniasis, and five controls. Each patient with NSV or trichomoniasis was studied before and after treatment with metronidazole.

Address for reprints: Dr M J Balsdon, Department of Genitourinary Medicine, St Mary's Hospital, Portsmouth, Hampshire PO3 6AD

Accepted for publication 15 March 1983

\section{Patients and methods}

SAMPLE COLLECTION

Five women with trichomoniasis and five with NSV associated with $G$ vaginalis ${ }^{45}$ were selected for study from patients attending the department of genitourinary medicine, St Mary's Hospital, Portsmouth. Ethical approval for the study was obtained from the appropriate hospital committee. The women were studied before and after treatment with metronidazole (Flagyl) $400 \mathrm{mg}$ given by mouth twice daily for five days. The clinical diagnoses were not made known to the laboratory until the end of the study. A further five women who had no genital infection or condition acted as controls.

Patients with trichomoniasis had genital inflammation, purulent vaginal discharge, and active trichomonads in the wet mount preparation. NSV associated with $G$ vaginalis was diagnosed on the basis of a smooth greyish white vaginal discharge and characteristic microscopy. The wet mount showed clue cells and rafts (floating clumps of coccobacilli) and the Gram stained smear showed masses of Gram variable small coccobacilli (the "pepper on salt" pattern). ${ }^{5}$ All study patients and controls had 
negative cultures for Neisseria gonorrhoeae and Candida spp and none had had contact with men with non-specific urethritis. Vaginal $\mathrm{pH}$ was measured by narrow range indicator paper.

A quantity of $2 \mathrm{ml}$ of sterile physiological saline $(0 \cdot 87 \%)$ was instilled into the vagina by syringe through a speculum. A sterile plastic loop was used to move any adherent vaginal secretions into the pooled fluid in the posterior fornix. The pooled fluid was then removed by sterile syringe and tubing and transferred to a glass tube. It was centrifuged in a bench centrifuge at $1500 \times g$ for three minutes and the supernate stored at $-20^{\circ} \mathrm{C}$.

\section{TEST MATERIALS}

Putrescine dihydrochloride, cadaverine dihydrochloride, tyramine hydrochloride, spermidine phosphate, spermine tetrachloride, agmatine sulphate, dansyl chloride (5-(dimethylamino)naphthalene-l-sulphonyl chloride), didansyl putrescine, and didansyl cadaverine were obtained from Sigma London Chemical Company Ltd. All other reagents were of analytical grade. Precoated plastic silica gel thin layer chromatography (TLC) plates (Polygram Sil G, $0.25 \mathrm{~mm}$ layer) were obtained from Camlab, Cambridge.

\section{ESTIMATION OF AMINES}

Amines in the vaginal samples were estimated as dansyl derivatives by a method similar to that described by other workers. ${ }^{67} \mathrm{~A} 200 \mu \mathrm{l}$ portion of the vaginal sample was taken and $10 \mu \mathrm{l}$ of agmatine sulphate ( $2 \mathrm{mg} / \mathrm{ml}$ aqueous solution) added as internal standard. The $\mathrm{pH}$ was adjusted to $\mathrm{pH} 9$ using $\mathrm{NaHCO}_{3}$ and $400 \mu \mathrm{l}$ dansyl chloride $(30 \mathrm{mg} / \mathrm{ml}$ in acetone) were added. The sample was left for 16 hours in the dark, excess dansyl chloride was removed by addition of aspartic acid, and the sample was left for a further hour in the dark. After adjustment to $\mathrm{pH} 11$ the dansylated amines were extracted into diethyl ether, the ether extract evaporated to dryness under reduced pressure, and the residue redissolved in $200 \mu \mathrm{l}$ chloroform. Five microlitres of this chloroform solution were applied to a TLC plate, which was then developed twice in the dark in cyclohexane and ethyl acetate (3 parts to 2), dried overnight in the dark, and sprayed with isopropanol and triethanolamine (4 parts to 1) to enhance and stabilise the fluorescence.

The fluorescence of the dansylated amines on the TLC plates was measured using a Perkin-Elmer MPF4 fluorescence spectrophotometer equipped with a plate scanner (excitation $365 \mathrm{~nm}$, emission $500 \mathrm{~nm}$ ). Dansylated standard amines were included on each TLC plate. Putrescine, cadaverine, and tyramine quantities were estimated by measuring their peak areas, together with that of the internal standard in each sample, and referring these to standard curves. All dansylations were carried out in duplicate and the mean value of the two results taken.

\section{MASS SPECTROMETRY}

Mass spectrometry was used to confirm the identities of the amines in two samples from each of the patients with NSV and trichomoniasis. A $400 \mu \mathrm{l}$ portion of vaginal sample was dansylated as described above and the final chloroform solution was banded on to a TLC plate. After development the fluorescent material from appropriate bands on the plate was extracted into chloroform and evaporated to dryness under reduced pressure.

Mass spectra were recorded on a VG MM 12 B mass spectrometer using electron impact conditions. An ionising potential of $40 \mathrm{eV}$ was used with a source temperature of $200^{\circ} \mathrm{C}$. The spectrometer was scanned repetitively over the range 520-620 amu. The presence of a peak at $\mathrm{m} / \mathrm{z} 554$ was taken as evidence for didansyl putrescine, a peak at $\mathrm{m} / \mathrm{z} 568$ as evidence for didansyl cadaverine, and a peak at $\mathrm{m} / \mathrm{z} 603$ as evidence for didansyl tyramine.

\section{STATISTICAL TESTS}

The mean cadaverine to putrescine ratios and the mean log (tyramine) values in the patients with NSV and trichomoniasis were compared by means of Student's $t$ test.

\section{Results}

The amounts of putrescine, cadaverine, and tyramine in vaginal washings from the patients and controls are summarised in the table. The characteristic TLC separation of the three amines is shown in the figure.

Controls-All five samples from the controls contained small amounts of putrescine and two of the five (2048 and 2173) small amounts of cadaverine and tyramine. These two patients had large quantities of bacteria other than lactobacilli on their Gram stained smears. The vaginal $\mathrm{pH}$ in the controls ranged from $3 \cdot 8$ to $4 \cdot 5$.

Untreated trichomoniasis-Four of the five samples from the untreated patients with trichomoniasis contained both putrescine and cadaverine, generally in greater quantities than in the controls. The fifth sample $(1587 / 1)$ contained a trace of putrescine only. In all cases the amount of putrescine was at least as high as that of cadaverine with a mean cadaverine to putrescine ratio of about $0 \cdot 8: 1$. Tyramine was detected in only two of the five samples in the group. The vaginal $\mathrm{pH}$ in these patients ranged from $5 \cdot 4$ to $6 \cdot 8$. 
TABLE Amine concentrations ( $\mu \mathrm{mol} / \mathrm{l})$ in vaginal samples from 15 women

\begin{tabular}{|c|c|c|c|c|}
\hline Group & Sample No* & Putrescine & Cadaverine & Tyramine \\
\hline Controls & $\begin{array}{l}2048 \\
2072 \\
2107 \\
2169 \\
2173\end{array}$ & $\begin{array}{r}69 \\
T \\
T \\
T \\
34\end{array}$ & $\begin{array}{r}97 \\
\text { ND } \\
\text { ND } \\
\text { ND } \\
\text { T }\end{array}$ & $\begin{array}{r}63 \\
\text { ND } \\
\text { ND } \\
\text { ND } \\
\mathbf{T}\end{array}$ \\
\hline $\begin{array}{l}\text { Patients with } \\
\text { trichomoniasis }\end{array}$ & $\begin{array}{r}986 / 1 \\
/ 2 \\
1073 / 1 \\
/ 2 \\
1523 / 1 \\
/ 2 \\
1587 / 1 \\
/ 2 \\
1592 / 1 \\
/ 2\end{array}$ & $\begin{array}{r}2420 \\
\text { ND } \\
166 \\
\text { ND } \\
767 \\
\text { ND } \\
T \\
162 \\
214 \\
T\end{array}$ & $\begin{array}{c}2020 \\
\text { ND } \\
136 \\
\text { ND } \\
755 \\
\text { ND } \\
\text { ND } \\
302 \\
55 \\
\text { ND }\end{array}$ & $\begin{array}{l}403 \\
\text { ND } \\
\text { ND } \\
\text { ND } \\
\text { ND } \\
\text { ND } \\
\text { ND } \\
86 \\
T \\
\text { ND }\end{array}$ \\
\hline $\begin{array}{l}\text { Patients with } \\
\text { NSV associated } \\
\text { with Gardnerella } \\
\text { vaginalis }\end{array}$ & $\begin{array}{r}761 / 1 \\
/ 2 \\
1074 / 1 \\
/ 2 \\
1086 / 1 \\
/ 2 \\
1165 / 1 \\
/ 2 \\
1967 / 1 \\
/ 2\end{array}$ & $\begin{array}{r}190 \\
\text { ND } \\
650 \\
\text { ND } \\
485 \\
T \\
46 \\
\text { ND } \\
82 \\
T\end{array}$ & $\begin{array}{r}146 \\
\text { ND } \\
1530 \\
\text { ND } \\
946 \\
\text { ND } \\
62 \\
\text { ND } \\
106 \\
\text { ND }\end{array}$ & $\begin{array}{r}64 \\
\text { ND } \\
576 \\
T \\
505 \\
T \\
\text { ND } \\
\text { ND } \\
35 \\
\text { ND }\end{array}$ \\
\hline
\end{tabular}

$* / 1=$ untreated; $/ 2=$ after metronidazole treatment; $T=\operatorname{trace}(10-30 \mu \mathrm{mol} / \mathrm{l}) ; \mathrm{ND}=$ not detected $(<10 \mu \mathrm{mol} / \mathrm{l})$.

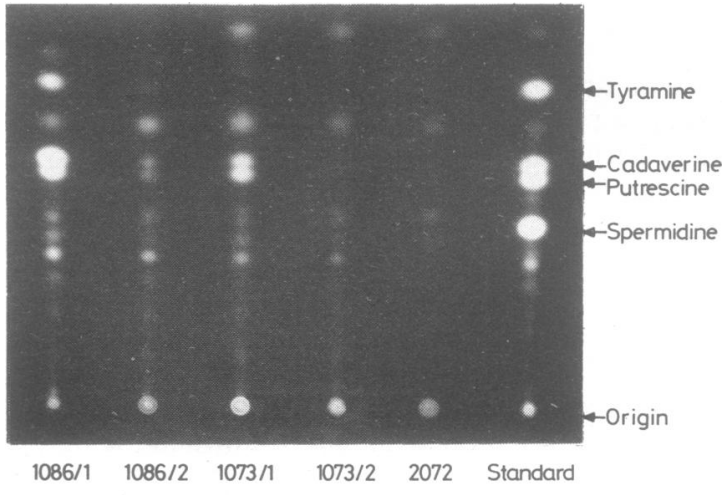

FIGURE Thin layer chromatographic separation of the dansylated amines in five vaginal samples.

Untreated NSV-All five samples from the untreated patients with NSV contained putrescine and cadaverine and four of them appreciable amounts of tyramine. The amounts of all three amines were generally much greater than those in the controls. Four of the five samples contained more cadaverine than putrescine, the mean cadaverine to putrescine ratio being $1 \cdot 5: 1$. The vaginal $\mathrm{pH}$ in these patients ranged from $5 \cdot 2$ to $5 \cdot 7$.

After metronidazole treatment-All the patients with trichomoniasis or NSV associated with $G$ vaginalis were cured after treatment with metronidazole. Only trace amounts of any of the three amines were detected in the vaginal samples from these treated patients, except sample 1587/2 (trichomoniasis group) which contained appreciable amounts of all three amines. At follow up the Gram stained smear of the vaginal secretions from this patient showed a mixed bacterial flora in contrast to the lactobacilli which were present in the other treated patients. The vaginal $\mathrm{pH}$ of the treated patients ranged from $4 \cdot 0$ to $4 \cdot 7$. The polyamines spermidine and spermine were generally present in all three groups of samples and were not affected by metronidazole treatment.

Statistical analysis-The $t$ test comparison of the mean cadaverine to putrescine ratios showed a significant difference between the patients with trichomoniasis and those with NSV $(t=2 \cdot 75 ; \mathrm{p}<0.05)$. The difference in amounts of tyramine between the two groups was not statistically significant $(t=1 \cdot 31$; $\mathrm{p}=\mathbf{0} \cdot 22$ ).

Spectrometry-The identities of the amines in the following samples were confirmed by mass spectrometry: 986/1 and 1523/1 (trichomoniasis group); 1074/1 and 1086/1 (NSV group).

\section{Discussion}

We have shown, as have Chen $e t a l,{ }^{3}$ that putrescine and cadaverine are present in vaginal washings from patients with trichomoniasis. Putrescine but not cadaverine was produced in large quantities by $T$ 
vaginalis grown in a modified Bushby's medium (unpublished data). Cadaverine may be found in vivo but not in Bushby's culture medium because its production depends on the presence of adequate amounts of lysine or on a particular $\mathrm{pH}$; alternatively, cadaverine may be produced in the vagina by organisms other than $T$ vaginalis. It is interesting that relatively more putrescine than cadaverine was produced in patients with trichomoniasis than in those with NSV associated with $G$ vaginalis. $T$ vaginalis may be primarily a putrescine producer but associated organisms may modify amine production in vivo. Tyramine was absent from most of the trichomoniasis samples but present in most of the NSV samples; the difference in amounts of tyramine between the two groups was not statistically significant, however, so larger numbers of samples should be examined to determine if this trend is genuine.

Our findings also confirm the reports of Chen et al that large amounts of putrescine and cadaverine are present in vaginal washings from patients with NSV, together with tyramine in some cases. ${ }^{13}$ The mean cadaverine to putrescine ratio in their study was 1.4:1 which compares with our figure of $1 \cdot 5: 1$. Chen et al suggested from their in vitro studies that putrescine and cadaverine were produced by metronidazole sensitive anaerobic organisms and tyramine by metronidazole resistant facultative organisms. ${ }^{1}$ In our study and theirs, however, production of all three amines in patients with NSV virtually ceased after metronidazole treatment. The difference between the clinical and laboratory results could be explained by the sensitivity of facultative anaerobes, such as $G$ vaginalis, to the 2-hydroxymethyl metabolite of metronidazole but not to metronidazole itself. ${ }^{8-10}$

It is interesting that all the women in whom only lactobacilli were noted on Gram stained smears of vaginal material (three controls and all the treated patients except for one with trichomoniasis) had only trace amounts of amines in their vaginal washings. The three women from these groups (two controls and one treated) with vaginal washings giving greater amounts of amines had Gram stained smears showing mixed bacteria, probably anaerobic, and no detectable lactobacilli. This suggests that amine production in the vagina is associated with organisms other than lactobacilli. Although the ability to decarboxylate amino acids to their corresponding amines is widespread in bacteria, it appears that production of any amine by a particular organism is greatly influenced by $\mathrm{pH}$ and substrate availability and possibly by other factors. ${ }^{11} 12$ Amine production is highest at acidic $\mathrm{pH}$ and its role is thought to be the neutralisation of acid products of carbohydrate fermentation. ${ }^{11} 12$

We thank the staff in the department of genitourinary medicine, St Mary's Hospital, Portsmouth for their help and Dr B J Slater of May \& Baker Ltd for performing mass spectrometry.

\section{References}

1. Chen KCS, Forsyth PS, Buchanan TM, Holmes KK. Amine content of vaginal fluid from untreated and treated patients with nonspecific vaginitis. J Clin Invest 1979;63:828-35.

2. Pheifer TA, Forsyth PS, Durfee MA, Pollock HM, Holmes KK. Nonspecific vaginitis-role of Haemophilus vaginalis and treatment with metronidazole. N Engl J Med 1978;298: 1429-34.

3. Chen KCS, Amsel R, Eschenbach DA, Holmes KK. Biochemical diagnosis of vaginitis: determination of diamines in vaginal fluid. $J$ Infect Dis 1982; 145:337-45.

4. Gardner HL. Haemophilus vaginalis vaginitis after twenty-five years. Am J Obstet Gynecol 1980; 137:385-90.

5. Balsdon MJ, Taylor GE, Pead L, Maskell R. Corynebacterium vaginale and vaginitis: a controlled trial of treatment. Lancet 1980; i: 501-4.

6. Wyatt GR, Rothaus K, Lawler D, Herbst EJ. Ornithine decarboxylase and polyamines in silkmoth pupal tissues: effects of ecdysone and injury. Biochim Biophys Acta 1973;304:482-94.

7. Gittins M, Cooke KB. The detection and determination of polyamines from human urine. Biochem Soc Trans 1978;6: 212-4.

8. Balsdon MJ, Jackson D. Metronidazole metabolite and Gardnerella vaginalis (Corynebacterium vaginale). Lancet 1981;i: 1112 .

9. Shanker S, Munro R. Sensitivity of Gardnerella vaginalis to metabolites of metronidazole and tinidazole. Lancet 1982; i: 167.

10. Easmon CSF, Ison CA, Kaye CM, Timewell RM, Dawson SG. Pharmacokinetics of metronidazole and its principal metabolites and their activity against Gardnerella vaginalis. $\mathrm{Br}$ $J$ Vener Dis 1982;58:246-9.

11. Gale EF. The bacterial amino acid decarboxylases. In: Nord FF, ed. Advances in Enzymology, vol VI. New York: Interscience Publishers Inc, 1946: 1-32.

12. Morris DR, Fillingame RH. Regulation of amino acid decarboxylation. Ann Rev Biochem 1974; 43:303-25. 